\title{
Çapraz Akışı ıaralel Borulu Boru Demetinde Entropi Üretiminin Analitik ve Deneysel Olarak İncelenmesi
}

\author{
Alper YILMAZ*1, Tuncay YILMAZ ${ }^{2}$ \\ ${ }^{1}$ Çukurova Üniversitesi, Mühendislik -Mimarlık Fakültesi, Otomotiv Mühendisliği Bölümü, \\ Adana \\ ${ }^{2}$ Osmaniye Korkut Ata Üniversitesi, Mühendislik Fakültesi, Makine Mühendisliği Bölümü, \\ Osmaniye
}

Geliş tarihi: 22.01.2016 Kabul tarihi: 14.03.2016

\section{Özet}

Sıcak atık gazlardan sıvıya 1Sı transferinde en çok kullanılan 1sı eşanjörlerinden biri de çapraz akışlı boru demeti ısı eşanjörleridir. Boru dışından genelde gaz ve boru içinden de sıvı akmaktadır. Son yıllarda 1S1 eşanjörlerinin boyutlandırılmasında en önemli kriter olarak minimum entropi üretimi esas alınmaktadır. Bu çalışmada, sabit duvar sıcaklığında boru dışından akan havanın 1sı transferinden ve basınç kaybından dolayı entropi üretiminin hesaplanması için gerekli eşitlikler çıkarılmış ve ölçülen değerlerden hem 1S1 transferi hem de basınç kaybından dolayı entropi üretimleri belirlenmiş ve bulunan değerler tartışılmıştır.

Anahtar Kelimeler: Isı eşanjörü, Boru demeti, Isı transferi, Basınç kaybı, Entropi üretimi

\section{Analytical and Experimental Investigation of Entropy Generation for an In-line Cross-flow Tube Bank}

\begin{abstract}
One of the most commonly used heat exchangers for waste heat recovery from hot flue gases is crossflow tube bank heat exchangers. Generally, gas flows outside of the tubes and liquid flows inside the tubes. In recent years, minimum entropy generation is taken as the most important criterion for the dimensioning of heat exchangers. In this study, equations are derived for the calculation of entropy generation due to both heat transfer from hot flue gases that flows outside of the tube bank and pressure losses at constant wall temperature. Generated entropies are determined both due to heat transfer and due to pressure losses and they are discussed.
\end{abstract}

Keywords: Heat exchanger, Tube bank, Heat transfer, Pressure loss, Entropy generation

\footnotetext{
Yazışmaların yapılacağı yazar: Alper YILMAZ, Mühendislik Mimarlık Fakültesi, Otomotiv Mühendisliği Bölümü, Adana.alpyil@cu.edu.tr
} 


\section{GíRiş}

Çapraz akışlı boru demeti isı eşanjörleri bilhassa 1S1 geri kazanımında en çok kullanılan 1S1 eşanjörlerinden biridir. $\mathrm{Bu}$ 1sı eşanjörlerinde gaz tarafı kirlilik oranı egzoz gazlarından olduğu gibi yüksek olduğundan kanatçıksız borular tercih edilmektedir.

Isı eşanjörlerinin optimum tasarımı için çeşitli yöntemler önerilmektedir [1, 2]. Bu yöntemlerde genel olarak verilen bir basınç kaybında ve 1sı aktarımında minimum ısı eşanjör kesit alanı [3-6] veya minimum 1sı eşanjör hacmi [7-10] hedef alınmaktadır. Bazılarında ise verilen bir basınç kaybı ve 1sı aktarımında minimum eşanjör ilk yatırım ve işletme maliyeti istenmektedir. [1]. Ancak termodinamik açıdan en uygun görülen 1S1 eşanjöründe 1sı transferi ve basınç kaybı açısından minimum entropi üretiminin hedeflenmesidir [11].

Bunun için bu çalışmada, hem ısı transferi hem de basınç kaybından dolayı entropi üretimi incelenmiştir.

\section{DENEY DÜZENEĞİ}

\subsection{Deney Düzeneğinin Şematik Görünümü}

Deney düzeneği Şekil 1'de şematik olarak gösterilmiştir. V vantilatörüyle dışarıdan emilen hava, atık hava ile çapraz akışlı levhalı bir 1sı eşanjörü olan E2 de ön 1sıtmaya tabi tutulmaktadır. $\mathrm{Bu}$ hava 1sınmış su ile de bir miktar daha ısıtılarak hava 1sitıcısı (HI)'nda istenen sicaklığa getirilerek deney eşanjörü (DE)'ne verilmektedir. Burada soğutma kulesinde soğutulmuş ve sabit bir sicaklıkta tutulmak için su 1sitıcısı (SI)'ndan geçirilen su havayı soğutmak için kullanılmaktadır. Buradan çıkan su DE deney eşanjörüne verilerek 1sitılmaktadır. Deney eşanjöründen çıkan hava, E2 eşanjöründe sıcaklığ 1 düşürüldükten sonra atmosfere atılmaktadır. Deney eşanjöründe 1sıtılan su da sıcaklığı E1 eşanjöründe düşürülmekte ve sonra da soğutma kulesi (SK) da sicaklığı tekrar düşürülerek, $\mathrm{P}$ pompası vasitasıyla su 1sitıcısına gönderilmektedir.

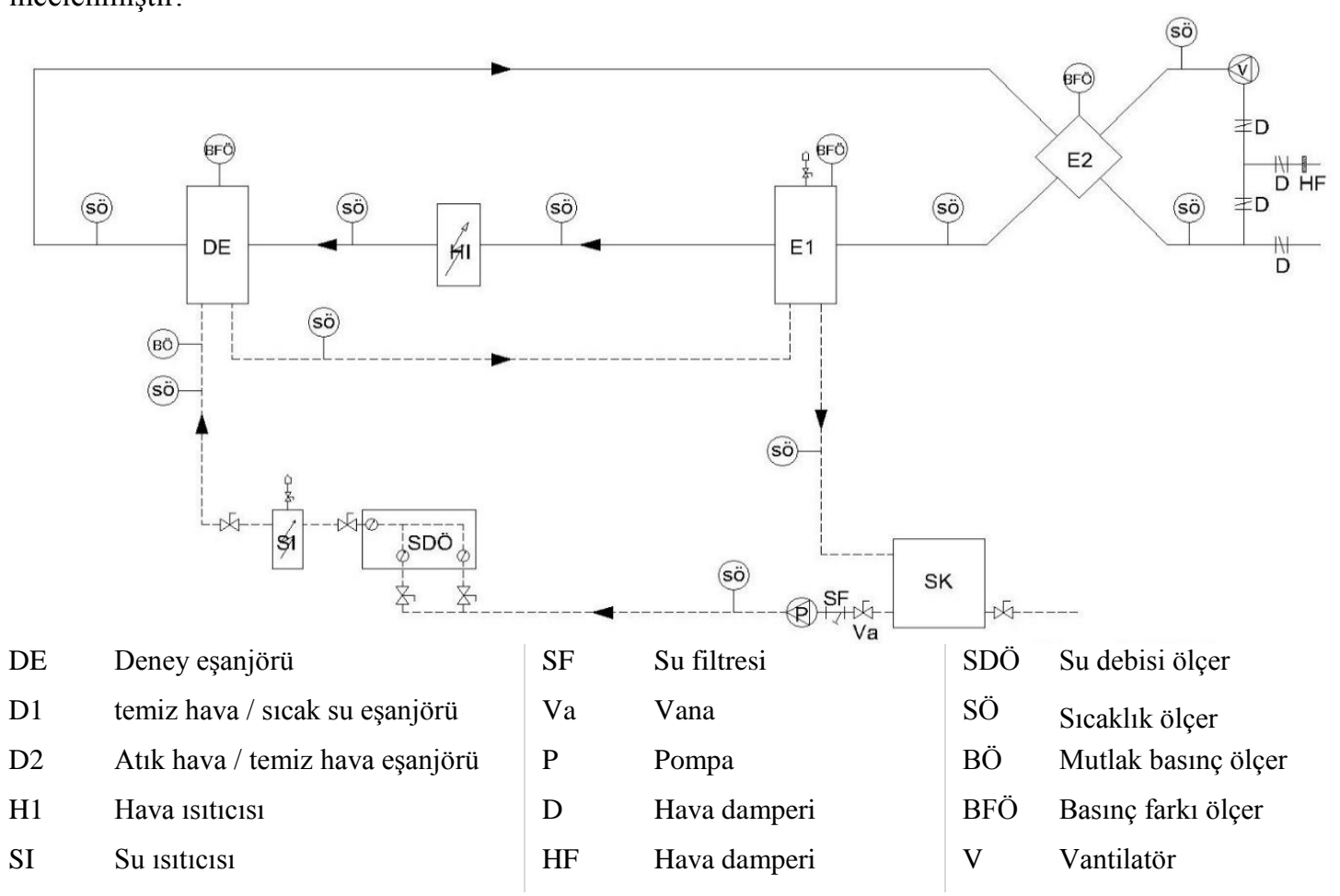

Şekil 1. Deney düzeneğinin şematik görünümü 
Deney düzeneği ve ölçme sistemleri hakkında ayrıntılı bilgi daha önceki bir çalışmada verilmiştir [13].

\subsection{Deney Eşanjörü}

Deney eşanjörü saptırılmış borular şeklinde olup, görünüm ve boyutları Şekil 2 ve 3'te gösterilmiştir.

\section{4. Ölçümler}

Sistemde tüm eşanjörler, hava ve su 1sitıcıları ile soğutma kulesi giriş ve çıkış sıcaklıklarını belirlemek için PT100 sensörleri kullanılmaktadır. Eşanjörlerin giriş ve çıkışlarında basınç farkları ölçülecek duruma getirilmiştir. Şekil 1'de deney düzeneği akış şeması üzerinde ölçüm noktaları gösterilmiştir.

Su debisi debi değerine göre rotametre 1, rotametre 2 ve elektronik debi ölçer ile ölçülmüştür. Bunların kalibrasyon eğrileri çıkarılmış olup, Şekil 4, 5 ve 6'da gösterilmiştir. Hava debisi ölçümü ise pitot tüpü yardımıyla gerçekleştirilmiştir. Buradaki ve deney eşanjöründeki basınç farkları basınç fark sensörleri ve data logger üzerinden bilgisayara aktarılmıştır.
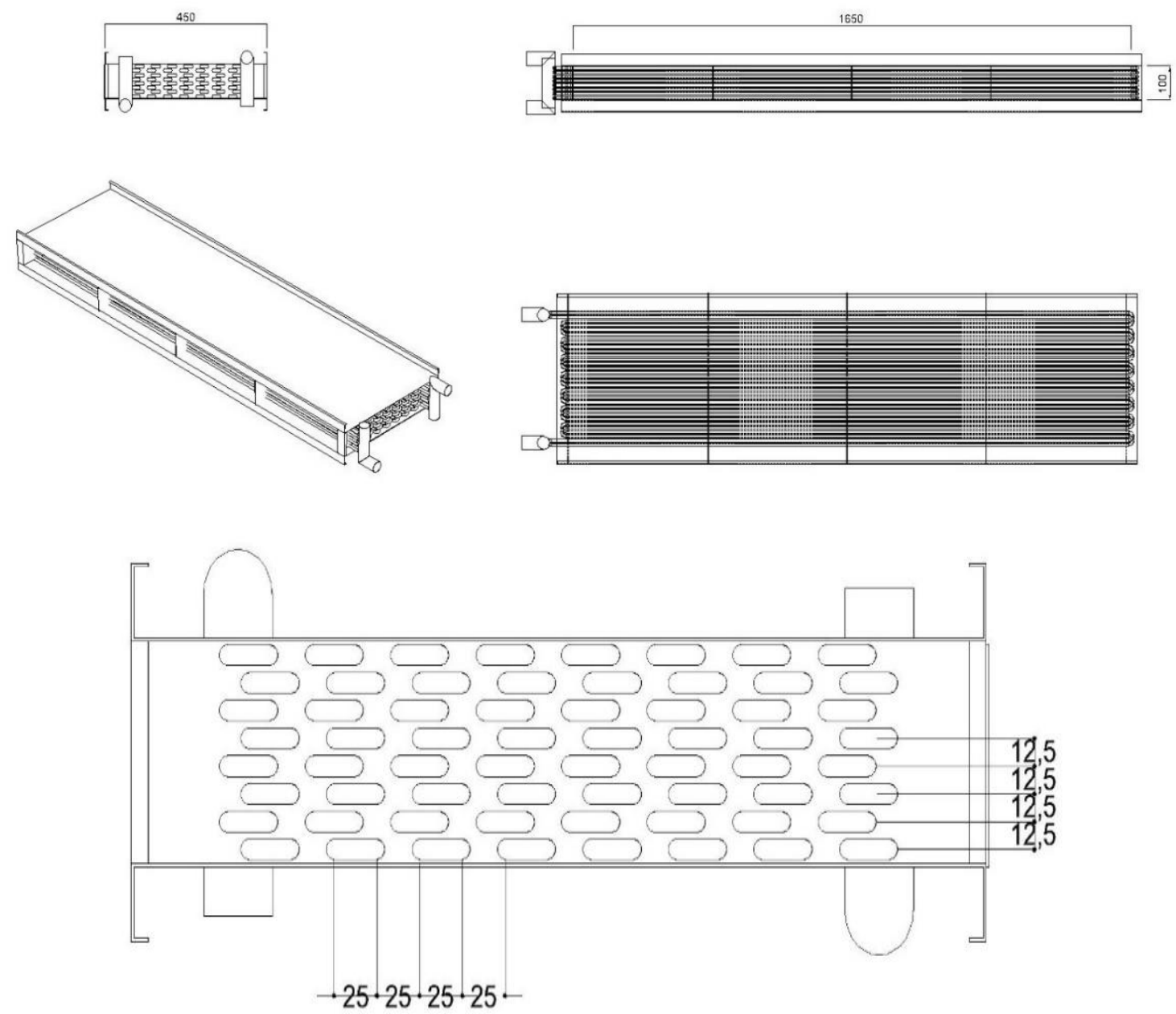

Şekil 2. Deney eşanjörünün (DE) görünümü ve boyutları 


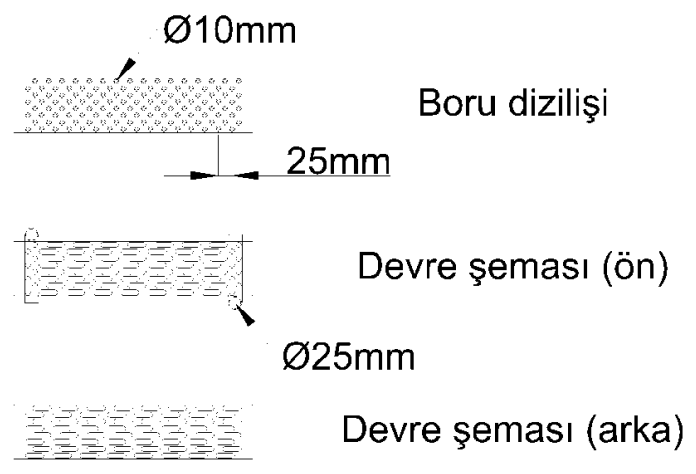

Şekil 3. Deney eşanjörünün (DE) boru dizilimi ve devre şeması

\section{DENEY DÜZENEĞINDE YAPILAN} ÖLÇÜMLERINN DEĞERLENDİRILMESI

\subsection{Transfer Edilen Isı ve Basınç Kaybının Belirlenmesi}

Deney eşanjöründe transfer edilen 1sı

$$
\begin{aligned}
& \dot{Q}_{h}=\dot{M}_{h} c_{p h}\left(T_{h g}-T_{h c ̧}\right) \\
& \dot{Q}_{s}=\dot{M}_{s} c_{p s}\left(T_{s c}-T_{s g}\right)
\end{aligned}
$$

Eşitlikleriyle belirlenmiştir. $\dot{M}_{h}$ ve $\dot{M}_{s}$ ölçülen su ve hava debileri olup, $c_{p h}$ ve $c_{p s}$ de

$$
\begin{aligned}
T_{h m} & =\frac{T_{h g}+T_{h c}}{2} \\
T_{s m} & =\frac{T_{s g}+T_{s c ̧}}{2}
\end{aligned}
$$

Şeklinde hesaplanan ortalama hava ve su sıcaklıklarında belirlenen havanın ve suyun özgül 1sılarıdır. Ölçülen sıcaklıklar yardımıyla $\dot{Q}_{h}$ ve
$\dot{Q}_{s}$ belirlenmiş ve transfer edilen isı $\dot{Q}$ olarak da bunların ortalaması alınmıştır:

$$
\dot{Q}=\frac{\dot{Q}_{h}+\dot{Q}_{s}}{2}
$$

Deney eşanjöründe basınç kaybı $\Delta P$ basınç fark ölçer tarafindan doğrudan ölçülmüştür. Deney eşanjöründe akış yönünde borular arası mesafe $S_{L}$ ve akışa dik yönde mesafe $S_{T}$ aşağıdaki şekilde $d$ boru dış çapı ile boyutsuz hale getirilmiştir:

$$
\begin{aligned}
& s_{T}^{*}=\frac{s_{T}}{d} \\
& s_{L}^{*}=\frac{s_{L}}{d}
\end{aligned}
$$

Deney eşanjöründe ilgili değerler aşağıda verilmiştir.

$$
\begin{aligned}
& \frac{u_{\max }}{u}=\frac{5}{3} ; N_{L}=32 ; N_{T}=16 ; s_{T}^{*}=2.5 \\
& ; s_{L}^{*}=1,25
\end{aligned}
$$

Burada $u_{\max }$ boru demeti içindeki en yüksek hava hızı, $u$ eşanjöre girmeden önceki borusuz eşanjördeki hava hızı, $N_{L}$ ve $N_{T}$ akış yönünde ve akışa dik yöndeki boru sayılarıdır.

\subsection{Entropi Üretiminin Hesaplanması}

Bir akışta entropi üretimi,

$\sum \dot{M}_{g} s_{g}-\sum \dot{M}_{c} s_{c}+\sum \frac{\dot{Q}}{T}+\dot{S}_{u ̈ r}=\Delta S$

eşitliğinden belirlenir. Burada g girişi ve ç de çıkışı sembolize etmektedir. Sürekli akışta $\Delta S=0$ olacağından, üretilen entropi, 


$$
\dot{S}_{u i r}=\sum \dot{M}_{\zeta} s_{\zeta}-\sum \dot{M}_{g} s_{g}-\sum \frac{\dot{Q}}{T}
$$

Eşitliğinden bulunmalıdır. Bu eşitlikte toplam yerine integral yazılarak,

$$
\dot{S}_{\ddot{u} r}=\dot{M}\left(s_{c}-s_{g}\right)-\int_{g}^{c} \frac{d \dot{Q}}{T}
$$

şekline gelir. Bu eşitliğin diferansiyel yazımı da,

$$
d \dot{S}_{\ddot{u} r}=\dot{M} d s-\frac{d \dot{Q}}{T}
$$

şeklindedir. Buradaki ds entropi farkı ve iç enerjiler aşağıda verilen eşitlikler yardımıyla hesaplanabilir. Burada akışkan, deney eşanjöründe de kabul edilebileceği üzere, ideal gaz olarak kabul edilmiştir.

$$
\begin{aligned}
& T d s=d u+p d v \\
& h=u+p v \\
& d u=d h-p d v-v d p \\
& T d s=d h-v d p \\
& d s=\frac{d h}{T}-\frac{v}{T} d p \\
& d s=\frac{d h}{T}-R \frac{d p}{p}
\end{aligned}
$$

Eşitlik 12 entegre edildiğinde ve ds için Eşitlik 18 kullanıldığında, Eşitlik 19 elde edilir:

$$
\dot{S}_{u i r}=\dot{M}\left[\int \frac{d h}{T}-R \int \frac{d p}{p}\right]-\int \frac{d \dot{Q}}{T_{d}}
$$

$\mathrm{Bu}$ eşitliğin entegrasyonundan da Eşitlik bulunur:

$$
\dot{S}_{\ddot{u} r}=\dot{M}\left[c_{p} \ln \frac{T_{\zeta}}{T_{g}}-R \ln \frac{P_{\zeta}}{P_{g}}-\int \frac{d \dot{Q}}{T_{d}}\right]
$$

Şekil 4'te deney eşanjöründeki hava ve su tarafındaki sıcaklık değişimleri şematik olarak gösterilmiştir.

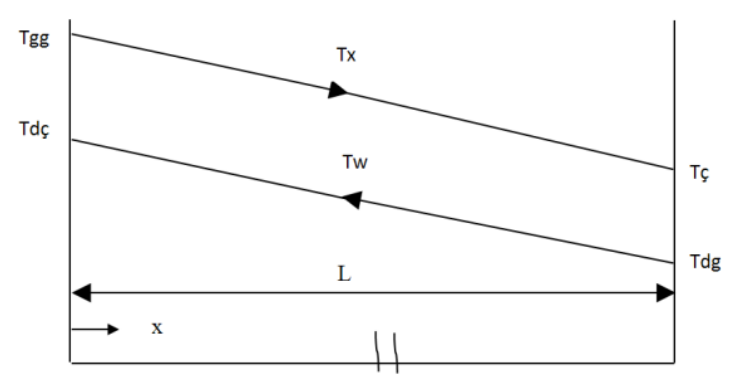

Şekil 4. Deney eşanjöründe gaz ve duvarın sıcaklık değişimi

Kullanılan 1sı eşanjöründe (DE) duvar sıcaklığı su sıcaklığına eşit alınabilir. Ayrıca eşanjör boyunca sıcaklık değişimi de lineer kabul edilebilir. Bu durumda duvar sıcaklığı için Eşitlik 21 yazılabilir:

$T_{d}=\left(1-x^{*}\right) T_{w i}+x^{*} T_{d c}$

Ayrıca 1Sı transferinden dolayı entropi üretimi için Eşitlik 22 geçerlidir.

$\dot{S}_{\dot{Q}}=-\int_{0}^{L} \frac{d \dot{Q}}{T_{d}}$

Eşanjör içinde 1S1 transferi katsayıs1, kolon sayısı $N_{T}$ çok olduğu için (16 adet), sabit kabul edilebilir. Böylece de Eşitlik 23 yazılabilir:

$d \dot{Q}=\dot{Q} \frac{d x}{L}=\dot{Q} d x^{*}$

Buradaki boyutsuz koordinat $x^{*}$ aşağıdaki gibi tarif edilmiştir:

$x^{*}=x / L$

Eşitlik 22 ve 23 'ten 
$\dot{S}_{\dot{Q}}=-\dot{Q} \int \frac{d x^{*}}{T_{d g}-x^{*}\left(T_{d g}-T_{d \zeta ̧}\right)}$

bağıntısı bulunur.

Buradan da

$$
\begin{aligned}
& \Delta T_{d}=T_{d g}-T_{d \zeta s} \\
& a=\frac{\Delta T_{d}}{T_{d g}}
\end{aligned}
$$

tanımlarıyla aşağıdaki eşitlikler elde edilir:

$$
\begin{gathered}
\dot{S}_{\dot{Q}}=-\frac{\dot{Q}}{T_{d g}} \int_{0}^{L} \frac{d x^{*}}{1-a x^{*}} \\
\dot{S}_{\dot{Q}}=-\frac{\dot{Q}}{T_{d g}} \frac{\ln (1-a)}{a} \\
\dot{S}_{\dot{Q}}=-\dot{Q} \frac{\ln \left(1-\frac{\Delta T_{d}}{T_{d g}}\right)}{\Delta T_{d}}
\end{gathered}
$$

$$
T_{d m}=\frac{T_{d g}-T_{d \varsigma}}{\ln \frac{T_{d g}}{T_{d c}}}
$$

Bu eşitliğe göre $T_{d m}$ değeri logaritmik ortalama bir duvar sıcaklığıdır. Bu değerlerle entropi üretimi Eşitlik 20'den aşağıdaki gibi elde edilir:

$$
\dot{S}_{\ddot{u} r}=\dot{M}_{h} c_{p} \ln \frac{T_{h \varsigma}}{T_{h g}}-R_{h} \ln \frac{P_{h \varsigma}}{P_{h g}}-\frac{\dot{Q}}{T_{d m}}
$$

Burada $R_{h}$ havanın gaz sabiti, $P_{h c}$ ve $P_{h g}$ de gazın eşanjöre giriş ve çıkış basınçlarıdır. Ayrica

$$
\dot{M}_{h} c_{p h}=\frac{\dot{Q}}{T_{g g}-T_{g c}}
$$

olduğundan

$$
\dot{S}_{\ddot{u} r}=\dot{Q} \frac{\ln \frac{T_{h \varsigma}}{T_{h g}}}{T_{g g}-T_{g \varsigma}}-\frac{\dot{Q}}{T_{d m}}=-\frac{\dot{Q}}{T_{g m}}-\frac{\dot{Q}}{T_{d m}}
$$

bulunur. Burada $T_{g m}$ logaritmik ortalama gaz Ortalama bir duvar sıcaklığı tarifi ile eşt. (31) yazılabilir:

$$
\dot{S}_{\dot{Q}}=-\frac{\dot{Q}}{T_{d m}}
$$

Burada ortalama duvar sıcaklığı aşağıdaki gibi tarif edilmiştir:

$$
T_{d m}=-\frac{\Delta T_{d}}{\ln \left(1-\frac{\Delta T_{d}}{T_{d g}}\right)}
$$




$$
P_{h \zeta}=P_{h g}-\Delta P
$$

olduğundan, Eşitlik 38 aşağıdaki duruma gelir:

$$
\dot{S}_{\ddot{u} r}=\dot{Q}\left(\frac{T_{g m}-T_{d m}}{T_{g m} T_{d m}}\right)-\dot{M} R \ln \frac{P_{h \xi}-\Delta P}{P_{h g}}
$$

Eşitlik 35 kullanılarak Eşitlik 40 aşağıdaki gibi yazılabilir:

$$
\dot{S}_{i i r}=\dot{Q}\left(\frac{T_{g m}-T_{d m}}{T_{g m} T_{d m}}\right)-\frac{\dot{Q}\left(1-\frac{1}{k}\right)}{T_{g g}-T_{g \xi}} \ln \left(1-\Delta P^{*}\right)
$$

Buradaki $k$ değeri izantropik gaz sabitidir. Entropi üretimi

$$
\dot{S}_{\ddot{u} r}=\dot{S}_{\ddot{u} r, q}+\dot{S}_{u ̈ r, p}
$$

Şeklinde 1sı transferi ve basınç kaybından dolayı olmak üzere ikiye ayrılabilir. Bunların tarifi aşağıda verilmiştir:

$$
\begin{gathered}
\dot{S}_{i \ddot{r}, q}=\dot{Q}\left(\frac{T_{g m}-T_{d m}}{T_{g m} T_{d m}}\right)=\dot{Q}\left(\frac{1}{T_{d m}}-\frac{1}{T_{g m}}\right) \\
\dot{S}_{\ddot{i r}, p}=-\dot{M} R \ln \left(1-\Delta P^{*}\right)=\frac{\dot{Q}\left(1-\frac{1}{k}\right)}{T_{g g}-T_{g \xi}} \ln \left(1-\Delta P^{*}\right)
\end{gathered}
$$

Birim transfer edilen 1sıya göre üretilen entropi de

$$
\begin{aligned}
& \frac{\dot{S}_{u r r}}{\dot{Q}}=\left(\frac{1}{T_{d m}}-\frac{1}{T_{g m}}\right)-\frac{1-\frac{1}{k}}{T_{g g}-T_{g \xi}} \ln \left(1-\Delta P^{*}\right) \\
& \dot{S}_{\ddot{u} r}=\dot{S}_{\ddot{u} r, q}+\dot{S}_{\ddot{u} r, p} \\
& \dot{S}_{\ddot{u} r, q}=\frac{1}{T_{d m}}-\frac{1}{T_{g m}}
\end{aligned}
$$

$\dot{s}_{i \ddot{u}, p}=-\frac{1-\frac{1}{k}}{T_{g g}-T_{g c}} \ln \left(1-\Delta P^{*}\right)$

Bu eşitliklerle $\dot{S}_{\ddot{u} r}, \dot{S}_{\ddot{u} r, q}, \dot{S}_{\ddot{u} r, p}$ ve bunların birim transfer edilen 1sıya göre özgül değerleri hesaplanabilir.

\subsection{Toplam ve Özgül Entropi Üretimi}

Şekil 5'te 1sı transferi ve basınç kaybından dolayı entropi üretimi $\dot{S}_{\ddot{u} r, q}, \dot{S}_{u ̈ r, p}$ ile toplam entropi üretimi $\dot{S}_{u ̈ r}$ eşanjör hava giriş hızının fonksiyonu olarak gösterilmiştir. Düşük hızlarda basınç kaybından dolayı entropi üretimi çok düşüktür. Eğrilerden bu entropi üretimlerinin ancak yaklaşık $5 \mathrm{~m} / \mathrm{s}$ hızında eşit olabilecekleri anlaşılmaktadır.

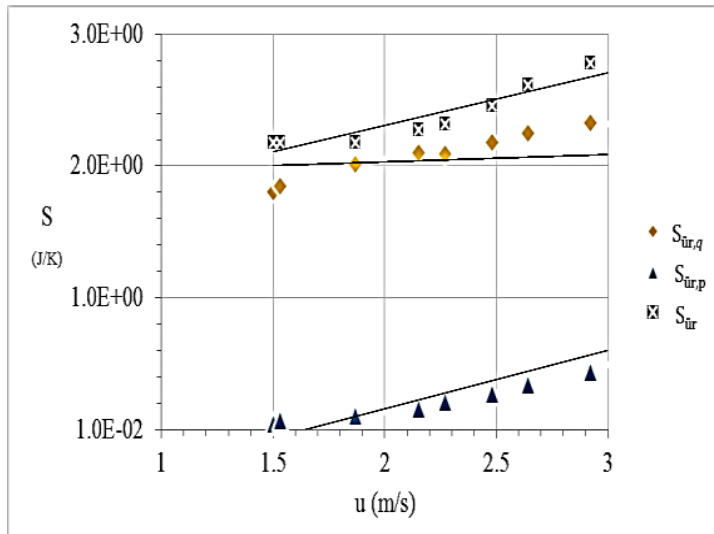

Şekil 5. DE'nde entropi üretiminin eşanjör hava giriş hızı ile değişimi

Şekil 6'da 1sı transferi ve basınç kaybından dolayı özgül entropi üretimi $\dot{s}_{\ddot{u} r, q}, \dot{s}_{\ddot{u} r, p}$ ile toplam özgül entropi üretimi $\dot{s}_{u ̈ r}$ eşanjör hava giriş hızının fonksiyonu olarak gösterilmiştir. Eğrilerden, özgül entropi üretimlerinin davranışlarının entropi üretimlerininkine benzer olduğu görülmektedir. 


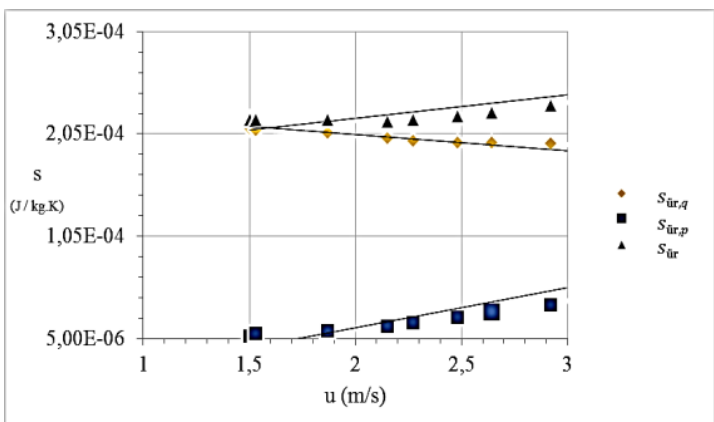

Şekil 6. DE’nde özgül entropi üretiminin eşanjör hava giriş hızı ile değişimi

\section{SONUÇLAR}

Çapraz akışlı paralel boru demeti 1sı eşanjöründe düşük hızlarda basınç kaybından dolayı entropi üretimi çok düşük olup 1sı transferinden dolayı gerçekleşen entropi üretimi toplam entropi üretiminde ciddi yer kaplamaktadır. Entropi üretimlerinin ancak yaklaşık $5 \mathrm{~m} / \mathrm{s}$ hızında eşit olabilecekleri anlaşılmaktadır. Özgül entropi üretimlerinin davranışlarının entropi üretimlerininkine benzer olduğu görülmektedir.

\section{TEŞEKKÜR}

$\mathrm{Bu}$ çalışma TEYDEB 7110772 nolu proje kapsamında hazırlanmış olup, Tübitak ve Barış Teknolojik Tesisat Sis. İnş. San. ve Tic. Ltd. şirketine katkılarından dolayı teşekkür ederiz.

\section{KAYNAKLAR}

1. Zukauskas, A., 1987. Convective Heat Transfer in Cross Flow, in Handbook of Single Phase Convective Heat Transfer, ed. S. Kakac, R. K. Shah, W. Aung, pp. 6/1-6/45,Wiley, New York.

2. Gaddis, E. S., 2010. Pressure Drop of Tube Bundles in Cross Flow, in VDI Heat Atlas, ed. VDI, pp. 1076-1091. Springer Verlag, BerlinHeidelberg.

3. Gnielinski, V., 2010. Heat Transfer in Cross Flow Around Single Rows of Tubes and Through Tube Bundles, in VDI Heat Atlas, ed. VDI, pp. 725-729. Springer Verlag, BerlinHeidelberg.
4. Bejan, A., E., Sciubba, E., 1992. The Optimal Spacing of Parallel Plates Cooled by Forced Convection, International Journal of Heat and Mass Transfer,vol. 35,pp. 3259-3264.

5. Yılmaz, A., Yılmaz, T., Büyükalaca, O., 2000. Optimum Shape and Dimensions of Ducts for Convective Heat Transfer in Laminar Flow at Constant Wall Temperature, International Journal of Heat and Mass Transfer.vol. 43, pp. 767-775.

6. Yilmaz, A., 2008. Optimum Length of Tubes for Heat Transfer in Turbulent Flow at Constant Wall Temperature, International Journal of Heat and Mass Transfer, vol. 51, pp. 3478-3485.

7. Yilmaz A., Yilmaz T., 2015. Optimum Design of Cross-Flow in-Line Tube Banks at Constant Wall Temperature, Heat Trasnfer Engineering, Accepted for Publication.

8. Y1lmaz, A., 2015. Dimensioning of Ducts for Maximal Volumetric Heat Transfer Taking Both Laminar and Turbulent Flow Possibilities into Consideration, Heat and Mass Transfer, Volume 51(4), 543-552.

9. Bejan, A., 1995. The Optimal Spacing for Cylinders in Crossflow Forced Convection, ASME Journal of Heat Transfer, vol. 117, pp. 767-770.

10. Fowler, A. J., Ledezma, G. A., and Bejan, A., 1997. Optimal Geometric Arrangment of Staggered Plates in Forced Convection, International Journal of Heat and Mass Transfer, vol. 40, pp. 1795-1805.

11. Matos, R. S. , Vargas, J. V. C. , Laursen, T. A., Saboya, 2001. F. E. M., Optimization Study and Heat Transfer Comparison of Staggered Circular and Elliptic Tubes in Forced Convection, International Journal of Heat and Mass Transfer, vol. 44, pp. 3953-3961.

12. Matos, R. S., Vargas, J. V. C., Laursen, T. A., Bejan, A., 2004. Optimally Staggered Finned Circular and Elliptic Tubes in Forced Convection, International Journal of Heat and Mass Transfer, vol. 47, pp. 1347-1359.

13. Muralikrishna, K., 2000. Heat Exchanger Design Targets for Minimum Area and Cost, Institution of Chemical Engineers, Trans. I ChemE, vol. 78, Part A, pp. 161-167. 